Published in final edited form as:

Crit Care Med. 2019 September ; 47(9): 1194-1200. doi:10.1097/CCM.0000000000003818.

\title{
ENABLERS AND BARRIERS TO IMPLEMENTING ICU FOLLOW- UP CLINICS AND PEER SUPPORT GROUPS FOLLOWING CRITICAL ILLNESS: THE THRIVE COLLABORATIVES
}

\author{
Kimberley J. Haines, PhD, BHSc (Physiotherapy), \\ Department of Physiotherapy, Western Health, Melbourne, Australia
}

Australian and New Zealand Intensive Care Research Centre, Monash University, Melbourne, Australia, +61466417689

Joanne McPeake, PhD, MSc, BN (Hons), RGN,

Glasgow Royal Infirmary, NHS Greater Glasgow and Clyde, Scotland, UK

School of Medicine, Dentistry and Nursing, University of Glasgow, Scotland, UK

Elizabeth Hibbert, B.Physiotherapy,

Department of Physiotherapy, Western Health, Melbourne, Victoria, Australia

Leanne M. Boehm, PhD, RN, ACNS-BC,

School of Nursing, Vanderbilt University, Nashville, Tennessee, US

Krishna Aparanji, MD, CPE,

Critical Care Medicine, Springfield Clinic, Springfield, Illinois, US

Rita N Bakhru, MD, MS,

Wake Forest University School of Medicine, Department of Internal Medicine, Section of

Pulmonary, Critical Care, Allergy and Immunology, Winston Salem, North Carolina, US

Anthony J Bastin, MBBS PhD,

Department of Peri-operative Medicine, St Bartholomew's Hospital, Barts Health NHS Trust,

London, UK

Sarah J Beesley, MD, MSc,

Pulmonary Division, Department of Medicine, Intermountain Medical Center, Murray, Utah, US

Center for Humanizing Critical Care, Intermountain Health Care, Murray, Utah, US

Pulmonary Division, Department of Medicine, University of Utah, Salt Lake City, Utah, US

Lynne Beveridge, RGN,

\footnotetext{
Corresponding author: Kimberley J. Haines PhD, BHSc (Physiotherapy), Department of Physiotherapy, Western Health, Sunshine Hospital, 176 Furlong Road, St Albans, Melbourne, Victoria 3021, Australia. Kimberley.haines@wh.org.au, Australian and New Zealand Intensive Care Research Centre, Monash University, Melbourne, Australia.

Author contributions: KH, JMP, LB, CS had full access to all of the data in the study and takes responsibility for the integrity of the data and the accuracy of the data analysis. All other authors contributed substantially to the study design, data analysis and interpretation, and the writing of the manuscript.

Summary conflict of interest statements: No conflicts of interest declared by other authors.

Publisher's Disclaimer: Disclaimer: This does not necessarily represent the views of the U.S. government or Department of Veterans Affairs.
} 
Glasgow Royal Infirmary, NHS Greater Glasgow and Clyde, Scotland, UK

Brad W Butcher, MD,

Department of Critical Care Medicine, University of Pittsburgh Medical Center, Pittsburgh,

Pennsylvania, US

\section{Kelly Drumright, MSN, RN, CNL,}

Tennessee Valley Healthcare System VA Medical Center, Nashville, Tennessee, US

Tammy L Eaton, MSN, RN, FNP-BC, ACHPN,

Palliative and Supportive Institute, UPMC Mercy, Pittsburgh, Pennsylvania, US

Thomas Farley, MS, ACNP,

School of Nursing, University of California San Francisco, San Francisco, California, US

Penelope Firshman, BSc,

Guy's and St Thomas' NHS Foundation Trust London, UK

Andrew Fritschle, PharmD,

Department of Pharmacy, Eskenazi Health, Indianapolis, Indiana, US

Clare Holdsworth, BPhys (Hons),

Department of Physiotherapy, Western Health, Melbourne, Victoria, Australia

Aluko A. Hope, MD, MSCE,

Division of Critical Care Medicine, Albert Einstein College of Medicine of Yeshiva University, New York, US

Annie Johnson, APRN, ACNP-BC,

CCRN Mayo Clinic, Rochester, Minnesota, US

Michael T. Kenes, PharmD, BCPS, BCCCP,

Department of Pharmacy, Wake Forest Baptist Medical Center, Winston Salem, North Carolina, US

Babar A. Khan, MD, MS,

Indiana University School of Medicine Research Scientist, Regenstrief Institute Inc. Indiana, US

Janet A. Kloos, RN, PhD, APRN-CCNS, CCRN,

University Hospitals Cleveland Medical Center, Cleveland, Ohio

Erin K. Kross, MD,

Department of Medicine, Division of Pulmonary, Critical Care and Sleep Medicine, Harborview Medical Center, University of Washington, Seattle, WA, US

Pamela Mactavish, BSc Pharm (Hons) MSc.,

Glasgow Royal Infirmary, NHS Greater Glasgow and Clyde, Scotland, UK

Joel Meyer, BM BCh DM,

Guy's \& St Thomas' NHS Foundation Trust, London, UK

Ashley Montgomery-Yates, MD,

Division of Pulmonary, Critical Care and Sleep Medicine, University of Kentucky, Kentucky, US 
Tara Quasim, MBChB, MD,

Glasgow Royal Infirmary, NHS Greater Glasgow and Clyde, Scotland, UK

Howard L. Saft, MD, MS,

Department of Medicine, Division of Pulmonary, Critical Care and Sleep Medicine, National Jewish Health, Denver, Colorado, US

Andrew Slack, MBBS, MRCP, EDIC, MD (Res),

Guy's \& St Thomas' NHS Foundation Trust, London, UK

Joanna Stollings, PharmD, FCCM, FCCP,

Department of Pharmaceutical Services, Vanderbilt University Medical Center, Nashville, Tennessee, US

Gerald Weinhouse, MD,

Department of Medicine, Division of Pulmonary and Critical Care, Brigham and Women's Hospital, Boston, Massachusetts, US

Jessica Whitten, PharmD,

Department of Pharmacy, Eskenazi Health, Indianapolis, Indiana, United States

Giora Netzer, MD, MSCE,

Division of Pulmonary and Critical Care Medicine, University of Maryland School of Medicine Department of Epidemiology and Public Health, University of Maryland School of Medicine, Maryland, US

Ramona O. Hopkins, PhD,

Department of Medicine, Pulmonary and Critical Care Division, Intermountain Medical Center, Murray, Utah, US

Center for Humanizing Critical Care, Intermountain Health Care, Murray, Utah, US

Psychology Department and Neuroscience Center, Brigham Young University, Provo, Utah, US

Mark E. Mikkelsen, MD, MSCE,

Division of Pulmonary, Allergy, and Critical Care Medicine, Hospital of the University of

Pennsylvania, Philadelphia, US

Theodore J Iwashyna, MD, PhD,

Department of Medicine, Division of Pulmonary \& Critical Care, University of Michigan, Ann

Arbor, Michigan, US

Center for Clinical Management Research, VA Ann Arbor Healthcare System, Ann Arbor, Michigan, US

Carla M. Sevin, MD

Department of Medicine, Division of Allergy, Pulmonary, and Critical Care Medicine, Vanderbilt University Medical Center, Nashville, Tennessee, US

\section{Abstract}

Objective: Data are lacking regarding implementation of novel strategies such as follow-up clinics and peer support groups, to reduce the burden of post-intensive care syndrome. We sought 
to discover enablers that helped hospital-based clinicians establish post-ICU clinics and peer support programs, and identify barriers that challenged them.

Design: Qualitative inquiry. The Consolidated Framework for Implementation Research was used to organize and analyze data.

Setting: Two learning collaboratives (ICU follow-up clinics and peer support groups), representing 23 sites, across three continents.

Subjects: Clinicians from 23 sites.

Measurement and Main Results: Ten enablers and nine barriers to implementation of ICU follow-up clinics were described. A key enabler to generate support for clinics was providing insight into the human experience of survivorship, to obtain interest from hospital administrators. Significant barriers included patient and family lack of access to clinics and clinic funding.

Nine enablers and five barriers to the implementation of peer support groups were identified. Key enablers included developing infrastructure to support successful operationalization of this complex intervention, flexibility about when peer support should be offered, belonging to the international learning collaborative. Significant barriers related to limited attendance by patients and families due to challenges in creating awareness, and uncertainty about who might be appropriate to attend and target in advertising.

Conclusions: Several enablers and barriers to implementing ICU follow-up clinics and peer support groups should be taken into account and leveraged to improve ICU recovery. Among the most important enablers are motivated clinician leaders who persist to find a path forward despite obstacles.

\section{Keywords}

critical illness; post-intensive care syndrome; peer support; intensive care unit; follow-up clinics

\section{INTRODUCTION}

Survivors of critical illness - both patients and their families - face a wide range of challenges (1-4). Despite a growing number of clinical trials designed to improve long-term outcomes after critical illness, post-intensive care syndrome and post-intensive care syndrome-family are all too common $(5,6)$. As care coordination for survivors is presently lacking, novel strategies have been called for to bridge the gap that survivors experience (7).

The Society of Critical Care Medicine's (SCCM) Thrive initiative implemented two international learning collaboratives (8) to facilitate rapid identification of strategies to improve outcomes for intensive care unit (ICU) survivors and their families. With the potential to reduce psychological distress among survivors and their families, improve care coordination, augment support, and facilitate recovery through optimized management and/or self-management, the Thrive post-ICU clinics collaborative (9) was launched in 2017, and the peer support collaborative $(7,10)$ in 2015.

It has been suggested that most new organizations fail, no matter what the field (11-13). This 'liability of newness' (12) can result from challenges in the work being attempted; the 
people trying to do the work; the organization set up to accomplish the work; the processes developed by that organization; or the institutional and international context in which that work is being attempted $(12,14)$.

We used qualitative inquiry with clinicians from 23 sites on three continents in the two collaboratives. We sought to discover enablers that helped hospital-based clinicians establish post-ICU clinics and peer support groups, and identify barriers that challenged them. We organized this investigation within the Consolidated Framework for Implementation Research (CFIR) (15) to insure systematic inquiry across the range of potential enablers and barriers.

\section{MATERIALS AND METHODS}

This study was reported using the Consolidated Reporting of Qualitative Research (COREQ) checklist (16).

\section{Setting and ethical approval}

Data were collected at the SCCM Congress (2018) where each collaborative held closed, daylong meetings. Two separate focus groups were conducted with each collaborative at these meetings. The institutional ethics committee of the principal investigator approved the study (Western Health Human Research and Ethics Committee 17/WH/170) and consent was implied through participation.

\section{Study design, participants, sampling, and recruitment}

Qualitative inquiry was used to provide an interpretative approach, describing complex phenomena that do not fit a quantitative model of hypothesis testing $(17,18)$. We used thematic analysis to identify themes from the data generated (19).

From the available 10 and 15 sites, for the clinic and peer support collaboratives respectively, we purposively sampled participants attending the in-person Congress meetings, representing various international sites (United States, United Kingdom, Australia) and professions (nursing, medical, allied health). We emailed collaborative members in advance of the focus groups and invited them to participate on the day of the meeting. Participants from collaborative sites not represented at the meetings, were purposively sampled and followed up by video-conferenced interview.

\section{Data collection and generation}

The researchers were experienced in qualitative methods and used a pre-designed set of semi-structured questions to guide the focus groups and interviews. Prompting questions were utilized to explore issues further. Participant demographic information was collected using a structured questionnaire. The data were audio-recorded and later professionally transcribed verbatim. The accuracy of the transcripts were verified against the audiorecordings and minor corrections made when necessary.

The CFIR was used to organize and analyze the data (15). The CFIR is widely used in implementation science and adaptable for use in different settings (20), comprising of five 
constructs (15): 1) intervention characteristics (refers to the intervention being implemented in an organization); 2) outer setting (refers to the economic, political, and social context within which the organization exists); 3 ) inner setting (refers to the structural, political, and cultural contexts through which implementation occurs); 4) characteristics of individuals (refers to the individuals involved in implementation); and 5) process (refers to the implementation process). The CFIR was well-suited to the process of implementing a post-ICU program within an institution. We used this framework in a table format, to help organize our data analysis. By grouping the data under the CFIR headings in this way, it allowed for easier visual representation of the data to highlight potentially important areas for future research and clinical implementation (21).

For the purposes of this study, peer support was defined as: 'services that bring together nonprofessionals with similar stressors or health problems for mutual support or unidirectional support from an experienced peer to a novice peer' (22) and where a previous description of six models (community based, psychologist-led outpatient, models-based within ICU follow-up clinics, online, groups based within ICU, and peer mentor models) were broadly applicable (23).

"ICU follow-up clinics were defined using a modified definition from a previous report (not limited to location): 'events that included at least one in-person clinic (or outpatient) encounter with a healthcare professional (specializing) in the treatment of ICU survivors' (9).

\section{Data analysis, researcher reflexivity, relationship with participants, and rigor}

Two researchers independently generated a list of possible of enablers and barriers from the data and organized them under the CFIR headings. The two researchers then worked together to categorize and code similar enablers and barriers to reduce duplication, and reordered data within the CFIR, developing major themes. These analyses were crossreferenced amongst the rest of the research team and against the original data. Final consensus on the themes was achieved through an iterative process. In order for the barriers and enablers to be classified as a 'key theme', they were identified through an iterative process and reaching consensus amongst the two researchers, and are therefore presented in no particular order, as they were described with equal importance.

The researchers are members of the Thrive collaboratives, hired by SCCM to conduct the evaluation and known to the participants through their prior involvement in the collaboratives. The researchers met regularly to discuss and address any issues as they arose throughout the study. To ensure rigor, regular crosschecking of analyses and data occurred within the research team. Member checking occurred during the participants' review of the manuscript as it is viewed as a critical technique for establishing the credibility of any qualitative study by reducing researcher bias and serves as a method of validating researcher interpretation $(24,25)$. 


\section{RESULTS}

Fifteen and eleven participants contributed data to the ICU follow-up and peer support clinic in-person focus groups, respectively. An additional two participants, representing a further two sites from the peer support collaborative, completed one-to-one interviews, with no response from the further two sites. Participant demographics are presented in Table 1. A cross-section of disciplines was sampled at both focus groups. A high proportion of the collaborative sites were represented (100\% for clinics and $87 \%$ for peer support). A collated high-level summary of key enablers and barriers is provided in Figure 1. Detailed enablers and barriers to the implementation of ICU follow-up clinics (tables 2 and 3) and peer support groups (tables 4 and 5) are presented within the CFIR, with supporting quotes.

\section{ICU Follow-Up Clinics}

We identified ten major themes categorized as enablers (Table E2) to the implementation of clinics: 1) interprofessional teamwork; 2) clinic design; 3) humanizing ICU survivorship; 4) alignment of ICU follow-up clinics to organizational priorities; 5) participation in SCCM Thrive Clinic Collaborative; 6) promotion of clinic; 7) obtaining funding; 8) motivated clinicians; 9) creative problem-solving to increase resources and efficiencies; and 10) streamlining clinic processes.

Humanizing survivorship was a major enabler interweaved across multiple constructs of CFIR. The concept of human connection between clinician and patient was a strong enabler within the clinics: "... build that rapport and sense of safety with them"(Participant 6), but was also used as an external strategy to engage other staff. Patient stories were used to motivate clinicians to participate in clinics, or obtain interest and funding from hospital administrators: “... you take the story of the patient and how the clinic helped them...to utilize them to motivate the hospital hierarchy..."(Participant 7).

Nine major themes categorized as barriers (Table E3) to the implementation of ICU followup clinics were identified: 1) staffing clinics; 2) identifying appropriate patients; 3) lack of collective identity of ICU survivorship; 4) patient and family limitations to accessing clinic; 5) lack of funding; 6) lack of space; 7) practice variation between clinicians; 8) limitations of clinicians as volunteers; and 9) hospital billing infrastructure.

Participants discussed patient and family limitations to accessing clinics as a major barrier. Access issues were largely related to practicalities of attending an in-person clinic, as one participant summarized: "caregivers have said it's just too much work to get that sick person, who's still so dependent for even basic things like dressing, bathing and mobility, to get them there..."(Participant 1). This also impacted caregiver employment: "they're losing time at work" (Participant 2). In addition to caregiver burden, other restrictions included distance from the clinic, particularly in rural settings, and the time and cost of attending another medical appointment.

Participants spoke at length about clinic funding as a major barrier. Funding appeared to cross between being described as a barrier: “... beg, borrow and steal the clinic time... since we had no funding..." (Participant 3 ) to becoming an enabler through creative problem- 
solving: "I figured out early there wasn't a lot of funding for post-ICU care if you called it that. But if you didn't call it that, people were more willing to pay for things"(Participant 4). Participants described the importance of external clinic funding and dependence on external funding to remain operational: "if we hadn't got the funding we would be dead in the water"(Participant 5).

\section{Peer Support Groups}

We identified nine major themes as enablers (Table E4) to implementing peer support groups: 1) building social cohesion; 2) defining operational processes; 3) accessing skilled group facilitation; 4) value of debriefing for the group facilitators; 5) membership to SCCM Thrive Peer Support Collaborative; 6) engaging participants into the group; 7) motivated interprofessional clinicians; 8) patient and family volunteers and advocates; 9) leveraging ICU follow-up clinics. Many of these enablers appeared to be of equal importance based on the volume of discussion generated and were clustered within the following three constructs of the CFIR:

1. 'Characteristics of the intervention' - building social cohesion, defining operational processes, accessing skilled group facilitation, and debriefing for the clinician group leads. Participants spoke about how groups run, operational systems and processes established, and the community of support created: "We've noticed just how effortless the groups are once you get people into them, because we were all worried, what are we going to talk about... but really the groups take care of themselves."(Participant 10)

2. 'The outer setting' - participants described membership to the SCCM Thrive Peer Support Collaborative as a crucial mechanism to provide each other with professional support during program implementation and served as a strong motivator: “...constant stimulation and motivation... really pushes the whole field forward."Participants described feeling they were not alone in challenges of implementation: "It's been helpful to hear that whether you're in the US, the $U K$, or Europe, or Australia, you face the same battles... it's nice to hear you're not alone, and struggling, because if you didn't, you'd probably give up a lot sooner:"(Participant 9)

3. 'The inner setting' - engaging participants into the group. Discussion focused on engaging patients and families to attend the groups. Strategies to facilitate attendance included use of email reminders, promotional materials, screening lists of patients discharged from the ICU, and provision of alternate ways to attend such as phone or video calls: "online conferencing technology has evolved so significantly... that probably would be something we could try."(Participant 14)

We identified five major themes categorized as barriers (Table E5): 1) patient and family non-attendance; 2) access to a skilled facilitator; 3) bureaucratic limitations of health services; 4) therapeutic trust and rapport; and 5) challenges in managing expectations of former patients as volunteers. 
Major barriers to implementing peer support groups were largely focused on the 'intervention characteristics' of the CFIR. Patient and family lack of attendance was a major barrier; contributing to a sense of limited success: "I would say we were largely unsuccessful in that we only had one patient show up."(Participant 8). Another related barrier was gaining access to a skilled group facilitator and "trying to decide who comes to facilitate and what their qualifications are"(Participant 9).

\section{DISCUSSION}

We describe, for the first time, enablers and barriers to implementing two promising strategies to facilitate ICU recovery for some patients and their families: follow-up clinics and peer support groups. A key enabler was using patient stories to create a human connection for other clinicians and hospital administrators in order to generate interest and support for ICU follow-up clinics. Significant barriers to ICU follow-up clinics included limitations to patients and families accessing clinics and funding to run them. Key enablers to setting up peer support groups included developing successful processes and systems to support the operationalization of the intervention, belonging to the international collaborative to provide momentum, and using strategies to engage patients and families to attend the group. Limited attendance by patients and families is a significant barrier to implementing peer support groups. Overall, despite the identification of varied and numerous barriers, there were equal, if not more, enablers, supporting implementation of these programs across multiple constructs of the CFIR.

Previous reviews have demonstrated that, while ICU follow-up clinics have existed for some time, there has been inadequate reporting of the interventions and overall the quality of the evidence is low $(9,26)$. Similarly, a recent systematic review demonstrated the evidence for peer support in critically ill populations is limited with a need for well-designed and rigorously reported research of this complex intervention (10). Previous thematic analysis has reported patient perspectives on follow-up clinics, indicating patients valued ICU follow-up although their care needs were not always met (27). Recent qualitative content analysis into the patients' perspective of ICU peer support groups has demonstrated value in creating a shared experience of survivorship (28).

However, a research gap exists in understanding clinicians' perspectives regarding implementation of follow-up clinics and peer support groups. Our study contributes important, complementary, and timely findings on the clinicians' perspective regarding key components required to support the start-up of these programs. These findings can be used to guide the design of future studies in this rapidly growing field as well as support clinical implementation. Further research is needed to explicitly understand how implementation of these programs differs across international settings, and how these themes may differ according to local context. This is particularly valuable in the broader healthcare context of an increasing population of ICU survivors (29) and the need to develop and test innovative models of care to improve long-term outcomes (30), where clinicians may be motivated by an ethical imperative to find new care approaches for survivors. Additionally, as local financial support is a common barrier, institutions engaging in risk-based strategies for cost 
containment, especially in the US where alternative payment models are being piloted, may want to consider supporting these interventions.

A limitation of this work is the lack of patient and family perspective regarding participation in these programs. We have future studies underway to specifically investigate the patient and family perspective regarding how these programs may have assisted in their recovery and what they viewed as essential components of a program.

A particular strength of this study is the international and interprofessional representation achieved to generate diversity in the data. The perspectives reported in this study were from a group of highly engaged and motivated professionals and may have been overly positive due to immersion in the field and time spent thinking, discussing and problem-solving within the learning collaboratives. These perspectives may differ across other clinicians who are working in the field, who were not sampled in this study. Another limitation, was the lack of representation of other key clinicians such as social workers and chaplains. However, our sample was representative of innovators who had spent time developing expertise, and therefore provided in-depth and nuanced data on the topic. Another strength is that we captured data during the stage of innovation and program start-up, yielding particularly rich early insights that may otherwise not be captured in different study designs. It should also be noted that data drawn from focus groups may have been influenced by group dynamics (31), however this is unlikely to have affected our analyses as we used simple thematic analysis.

If considering beginning a program, a key ingredient is a motivated clinician champion who can lead an interprofessional team to persist in finding a path forward despite obstacles and supportive administrators to help overcome bureaucratic "red tape" that might hinder success. Further, membership in the international collaboratives was perceived as an important element of success, providing an action-oriented community to drive improvements.

As in most programs, efficient processes are key. Our findings suggest that particularly critical processes include clearly defining how the intervention should be delivered and captured within a standard operating procedure. Such standardization also facilitates structured data collection as a part of routine practice to drive quality improvement.

And, finally, there are potentially modifiable barriers that should be pre-emptively mitigated. Particularly common and crosscutting seemed to be access to and sustainability of funding, skilled clinicians to staff these programs, identifying those survivors who might benefit and mechanisms to support their attendance.

Despite regional differences in processes of care, the key themes were consistent between countries. At a macro level, establishing these programs represents a future opportunity to undertake international comparative effectiveness research across varied healthcare settings to evaluate the impact on patient and family outcomes. At a meso and micro level, further exploration of different modes of program delivery should be tested that leverage advances in technology and reduce barriers to access, such as online platforms to host peer support groups and clinics delivered via a telemedicine model. 


\section{CONCLUSIONS}

There were several enablers and barriers to implementing ICU follow-up clinics and peer support groups. These timely findings provide new insights into the challenges, potential solutions and opportunities for designing, implementing, and testing such programs to support patients and families in their recovery.

\section{Supplementary Material}

Refer to Web version on PubMed Central for supplementary material.

\section{ACKNOWLEDGMENTS}

The authors would like to acknowledge and thank the following individuals who collectively contributed to the review of the original grant application: Dr Daniela Lamas, Dr James C. Jackson, Ms Belinda MacLeod-Smith, Ms Kate Cranwell, Dr Craig French, Dr Carol Hodgson.

Sources of support: K Haines, J McPeake, L Boehm, C Sevin are currently receiving funding from SCCM to undertake this work, although the supporting source had no input into the design, data collection and analysis, although approved the final manuscript for submission for publication. L Boehm is funded by NIH/NHLBI (1K12HL137943-01). The funding source reviewed and approved the manuscript for submission.

\section{REFERENCES}

1. Cuthbertson BH, Roughton S, Jenkinson D, et al. Quality of life in the five years after intensive care: a cohort study. Critical care 2010;14(1):R6. [PubMed: 20089197]

2. McPeake JHE, Christie L, Drumright K, Haines K, Hough T, Meyer J, Wade D, Andrews A, Bakhru RN, Bates S, Barwise J, Bastarache JA, Beesley SJ, Boehm LM, Brown S, Clay A, Firshman P, Greenberg SB, Harris W, Hill C, Hodgson C, Holdsworth C, Hope AA, Hopkins RO, Howell D, Janssen A, Jackson JC, Johnson A, Kross EK, Lamas D, MacLeod-Smith B, Mandel R, Marshall J, Mikkelsen ME, Nackino M, Quasim T, Sevin CM, Slack A, Spurr R, Still M, Thompson C, Weinhouse G, Wilcox ME, Iwashyna TJ. Models of Peer Support to Remediate Post-Intensive Care Syndrome: A Report Developed by the SCCM International Peer Support Collaborative (THRIVE) Critical Care Medicine (In press).

3. Haines KJ, Denehy L, Skinner EH, et al. Psychosocial outcomes in informal caregivers of the critically ill: a systematic review. Critical care medicine 2015;43(5):1112-1120. [PubMed: 25654174]

4. Herridge MS, Tansey CM, Matte A, et al. Functional disability 5 years after acute respiratory distress syndrome. The New England journal of medicine 2011;364(14):1293-1304. [PubMed: 21470008]

5. Maley JH, Brewster I, Mayoral I, et al. Resilience in Survivors of Critical Illness in the Context of the Survivors' Experience and Recovery. Annals of the American Thoracic Society 2016;13(8): 1351-1360. [PubMed: 27159794]

6. Marra A, Pandharipande PP, Girard TD, et al. Co-Occurrence of Post-Intensive Care Syndrome Problems Among 406 Survivors of Critical Illness. Critical care medicine 2018;46(9):1393-1401. [PubMed: 29787415]

7. Mikkelsen ME, Jackson JC, Hopkins RO, et al. Peer Support as a Novel Strategy to Mitigate PostIntensive Care Syndrome. AACN advanced critical care 2016;27(2):221-229. [PubMed: 27153311]

8. Medicine SoCC. THRIVE Peer Support Collaborative and Post ICU Clinic Collaborative. 2017 [cited 20189 May]Available from: http://www.sccm.org/Research/Quality/thrive/Pages/default.aspx

9. Lasiter S, Oles SK, Mundell J, et al. Critical Care Follow-up Clinics: A Scoping Review of Interventions and Outcomes. Clinical nurse specialist CNS 2016;30(4):227-237. [PubMed: 27309787] 
10. Haines KJB S; Hopkins R; McPeake J; Quasim T; Ritchie K; Iwashyna TJ Peer Support in Critical Illness: A Systematic Review Critical Care Medicine (in press) 2018.

11. Freeman JC GR; Hannan MT The liability of newness: Age dependence in organizational death rates. American Sociological Review 1983;48(5):692-710.

12. Stinchcombe AL. Social Structure and Organizations In: March JG, editor. Handbook of Organizations. New York: Routledge; 1965.

13. Yang T, Aldrich HE. "The liability of newness" revisited: Theoretical restatement and empirical testing in emergent organizations. Social science research 2017;63:36-53. [PubMed: 28202155]

14. Bruderi JaS R. Organizational Mortality: The Liabilities of Newness and Adolescence. Administrative Science Quarterly 1990;35(3):530-547.

15. Damschroder LJ, Aron DC, Keith RE, et al. Fostering implementation of health services research findings into practice: a consolidated framework for advancing implementation science. Implementation science: IS 2009;4:50. [PubMed: 19664226]

16. Tong A, Sainsbury P, Craig J. Consolidated criteria for reporting qualitative research (COREQ): a 32-item checklist for interviews and focus groups. International journal for quality in health care: journal of the International Society for Quality in Health Care / ISQua 2007;19(6):349-357.

17. Polkinghorne D Language and Meaning: Data collection in Qualitative Research. Journal of Counseling Psychology 2005;52(2):137-145.

18. Hammarberg K, Kirkman M, de Lacey S. Qualitative research methods: when to use them and how to judge them. Human reproduction 2016;31(3):498-501. [PubMed: 26759142]

19. Braun VaC V. Using thematic analysis in psychology. Qualitative Research in Psychology 2006;3(2):77-101.

20. Team CFfIR. Consolidated Framework for Implementation Research Constructs. 2014 [cited 2018 15 June]Available from: http://www.cfirguide.org/constructs.html

21. Keith RE, Crosson J, O’Malley A, Cromp D and Taylor E Using the Consolidated Framework for Implementation Research (CFIR) to produce actionable findings: a rapid-cycle evaluation approach to improving implementation. Implementation Science 2017;12(15).

22. Haines KJ, Beesley SJ, Hopkins RO, et al. Peer Support in Critical Care: A Systematic Review. Critical care medicine 2018;46(9):1522-1531. [PubMed: 29957717]

23. McPeake J, Hirshberg EL, Christie LM, et al. Models of Peer Support to Remediate Post-Intensive Care Syndrome: A Report Developed by the Society of Critical Care Medicine Thrive International Peer Support Collaborative. Critical care medicine 2019;47(1):e21-e27. [PubMed: 30422863]

24. Polit DFaB C.T. Essentials of Nursing Research: Appraising Evidence for Nursing Practice 7th ed Philadelphia, US: Lippincott, Williams and Wilkins; 2009.

25. Robson C Real World Research 3rd ed Oxford, UK: American Psychiatric Press; 2011.

26. Jensen JF, Thomsen T, Overgaard D, et al. Impact of follow-up consultations for ICU survivors on post-ICU syndrome: a systematic review and meta-analysis. Intensive care medicine 2015;41(5): 763-775. [PubMed: 25731633]

27. Prinjha S, Field K, Rowan K. What patients think about ICU follow-up services: a qualitative study. Critical care 2009;13(2):R46. [PubMed: 19338653]

28. Backman CG, Ahlberg M, Jones C, et al. Group meetings after critical illness-Giving and receiving strength. Intensive \& critical care nursing 2018.

29. Iwashyna TJ, Cooke CR, Wunsch H, et al. Population burden of long-term survivorship after severe sepsis in older Americans. Journal of the American Geriatrics Society 2012;60(6):1070-1077. [PubMed: 22642542]

30. Herridge MS. Long-term outcomes after critical illness: past, present, future. Current opinion in critical care 2007;13(5):473-475. [PubMed: 17762221]

31. Kitzinger $\mathbf{J}$ The methodology of focus groups: The importance of interaction between research participants. Sociology of Health and Illness 1994;16(1):103-121. 


\section{ENABLERS TO LEVERAGE}

Interprofessional teamwork

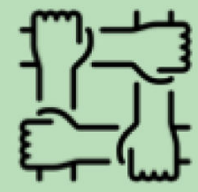

SCCM Thrive Collaboratives

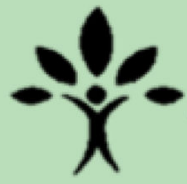

Defined operational processes

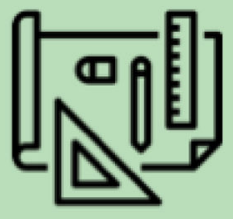

Motivated clinicians

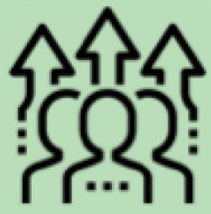

Human connection

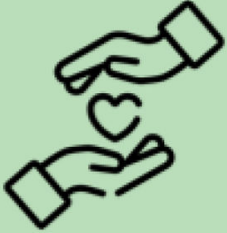

Creative problem-solving

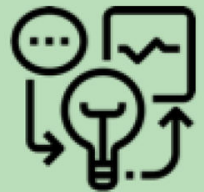

\section{BARRIERS TO PRE-EMPT}

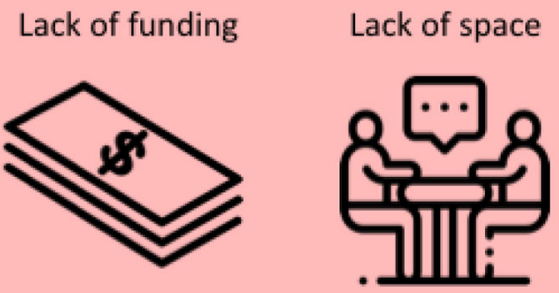

Identifying appropriate patients

Patient \& family attendance
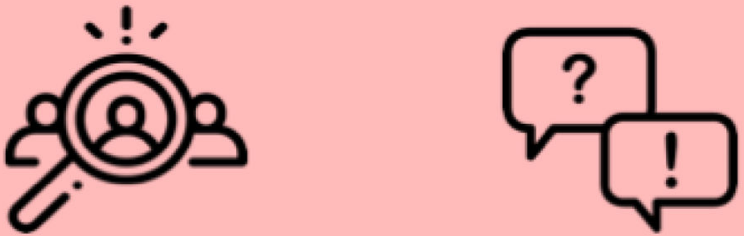

Figure 1 -.

Summary of Key Enablers and Barriers to Implementing ICU Follow-Up Clinics and Peer Support Programs 
Table 1 -

\section{Participant Demographics}

\begin{tabular}{lll}
\hline Variables & ICU Follow-Up Clinic (n=15) & Peer Support (n=13) \\
\hline Age (years) Mean (SD) & $41.3(8.6)$ & $44.1(8.7)$ \\
Gender, n (\%) male & $7(46.7)$ & $3(23.1)$ \\
Years of practice in critical care (years) Mean (SD) & $11.1(6.9)$ & $16.3(10.4)$ \\
Discipline, n (\%) & & \\
$\quad$ Medical & $8(53.3)$ & $6(46.2)$ \\
$\quad$ Nursing & $2(13.3)$ & $4(30.8)$ \\
$\quad$ Pharmacist & $4(26.7)$ & $1(7.7)$ \\
$\quad$ Allied Health & $1(6.7)$ & $2(17.4)$ \\
Sites represented, n (\%) of total collaborative sites & $10(100)$ & $13(87)^{*}$ \\
\hline
\end{tabular}

* 13 out of 15 available sites were sampled, with no response for follow-up interview from the remaining 2 sites. 